\title{
SITZUNGSBERICHTE DER SÄCHSISCHEN AKADEMIE DER WISSENSCHAFTEN ZU LEIPZIG
}

\section{MATHEMATISCH-NATURWISSENSCHAFTLICHE KLASSE}

\section{Band 110}

Heft 1 Prof. Dr.-Ing. Dr. h. c. PAUL GöRlich, tber die Laser und ihre Anwendung

1972. 24 Seiten $-8^{\circ}-$ M 2,30

Heft 2 Prof. Dr. Hasso EssBach, Zum Problem der Tumoren im Kindesalter

$$
\text { 1972. 24 Seiten - } 11 \text { Abbildungen auf } 10 \mathrm{Kunstdrucktafeln}-8^{\circ}-\mathrm{M} 6 \text {, - }
$$

Heft 3 Prof. Dr. med. WALTER BREDNow, Zur Anthropologie des Schwindels

1973. 17 Seiten -2 Abblidnngen auf 2 Kunstdrucktafeln $-8^{\circ}-$ M 2,50

Heft 4 Prof. Dr.-Ing. Dr. h. c. PAUL GöRLIch, Betrachtungen über den Wissenschaftlichen Gerătebau 1972. 39 Seiten $-8^{\circ}-$ M $3,-$

Heft 5 Prof. Dr. ERIOH RAMmLER, Einige Betrachtungen über Erdgas

$$
\text { 1974. } 43 \text { Seiten }-8 \text { Abbildungen }-3 \text { Tabellen }-8^{\circ}-\mathrm{M} 4,50
$$

Heft 6 Prof. Dr. Gustav E. R. SchULZE, Zur Rolle des Einfachheitsprinzips im physikslischen Weltbild 1974. 23 Seiten - 4 Abbildungen $-8^{\circ}-$ M 2,50

Heft 7 Prof. Dr. med. RoLf Emsrich, Zwischen Leben und Tod. Ärztliche Probleme der Thanatologie

1974. 22 Seiten -2 Abbildungen -4 Tabellen $-8^{\circ}-$ M 3,50

\section{Band 111}

Heft 1 Prof. Dr. Wilhely Marer, Vom Erbe Bernhard Riemanns 1975. 16 Seiten $-8^{\circ}-$ M 2,50

Heft 2 Prof. Dr. med. HANs Drischel, Organismus und geophysikalische Umwelt

$$
\text { 1975. } 50 \text { Seiten - } 25 \text { Abbildungen }-1 \text { Tabelle }-8^{\circ}-\text { M 7, - }
$$

Heft 3 Prof. Dr. Maria Hasse, Zum Begriff des allgemeinen Produkts von Kategorien 1975. 32 Seiten $-8^{\circ}-$ M 5,-

Heft 4 Prof. Dr.-Ing. Dr. h. c. KURT Schwabe, Analytische Probleme des Umweltschutzes.

$$
\text { 1975. } 28 \text { Seiten - } 9 \text { Abbildungen - } 2 \text { Tabellen }-8^{\circ}-\text { M 3,50 }
$$

Heft 5 Prof. Dr. Wolfgang Buchirgm, Die kopernikanische Wende und die Gravitation

$$
\text { 1975. 36 Seiten - } 2 \text { Farbtafeln }-8^{\circ}-\text { M 5,- }
$$

Heft 6 Prof. Dr. HrRmann Berg, Photopolarigraphie und Photodynamic 1975. 19 Seiten -2 Abbildungen -2 Tabellen $-8^{\circ}-$ M 3,-

Heft 7 Prof. Dr. MaNFred Gkrsch, Probleme der Insektizide aus heutiger Sicht 1976. 36 Seiten - 9 Abbildungen - 2 Tabellen $-8^{\circ}-\mathrm{M}_{4},-$

Band 112

Heft 1 Prof. Dr. WALter Brkdnow; Spiegel, Doppelspiegel und Spiegelungen - eine ,wunderliche Symbolik" Goethes 1975. 28 Seiten -4 Abbildungen $-8^{\circ}-\mathrm{M} 3,-$

Heft 2 Prof. Dr. Artor Lösche, ther negative absolute Temperaturen. Eine Einflihrung 1976. 26 Seiten - 12 Abbildungen - $8^{\circ}-$ M 4,-

Heft 3 Prof. Dr. med. Herbert JoRdan, Kurorttherapie: Prinzip und Probleme

$$
\text { 1976. } 31 \text { Seiten }-10 \text { A bbildungen - } 1 \text { Tabelle }-8^{\circ}-M_{4,50}
$$

Heft 4 Prof. Dr. FrIEdrioh Wolf / Dr. PeTER FröHIIoH, Zur Druckabhängigkeit von Ionenaustauschreaktionen 1977. 13 Seiten -6 Abbildungen -1 Tabelle $-8^{\circ}-$ M 2,-

Heft 5 Prof. Dr. Dietrich Uhumann, Möglichkeiten und Grenzen einer Regenerierung geschädigter $\mathrm{Oko}$ systeme 1977. 50 Seiten -20 Abbildungen -2 Tabellen $-8^{\circ}-$ M 6,50

Heft 6 Prof. Dr. Erich RAmmLer, Zwei Jahrzehnte Entwicklung des Einsatzes der Energietrăger Kohle und Frdōl im Weltmaßstab 1977. 29 Seiten -6 Abbildungen -4 Tabellen $-8^{\circ}-\mathrm{M}_{4,-}$

Heft 7 Prof. Dr. Ulrich FrerMoth, Umweltprobleme in der Ernăhrung 1977. 32 Seiten -3 Abbildungen -4 Tabellen $-8^{\circ}-$ M 4,- 